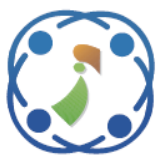

\title{
An Enhanced Framework for Sign Gesture Recognition using Hidden Markov Model and Adaptive Histogram Technique
}

\author{
Rajesh Kaluri ${ }^{1 *}$ \\ Pradeep Reddy $\mathrm{CH}^{1}$ \\ ${ }^{1}$ School of Information Technology and Engineering, VIT University, Vellore, India. \\ *Corresponding author's Email: rajesh.kaluri@vit.ac.in
}

\begin{abstract}
Gesture based communication is the fundamental method for correspondence for those with hearing and vocal incapacities. Communication via gestures comprises of making shapes or developments with human hands as for the head or other body parts. In this paper, we propose a new framework for recognizing sign gestures by using Hidden Markov Model (HMM) and Histogram based methods. Initially, the noise of an image will be eliminated by Wiener Filter and the image will be segmented with the help of Histogram oriented methods - Adaptive Histogram technique and then features will be extracted. The extracted features will be given to the HMM for training and recognition of gestures. Our experimental results show a better performance in terms of recognizing gestures from a blurred image compared to the existing segmentation methods.
\end{abstract}

Keywords: Sign Language Recognition, Adaptive Histogram Technique, Hidden Markov Model.

\section{Introduction}

Gesture Recognition empowers people to speak with the machine and associate normally with no mechanical gadgets. People speak with each other utilizing discourse, hand motions, outward appearances, and gesture based communication. It appears to be regular to utilize a similar kind of cooperation when speaking with PCs. This gets to be all the more critical for people tested with hearing and discourse disabilities $[1,2]$. Previously, scientists have proposed PC vision-based strategies for translating human motions taken from the video cameras. Gestures can start from any substantial movement or state however usually begin from the face or hand. Current concentrations in the field incorporate feeling acknowledgment from face and hand motion recognition $[3,4,5]$.

The objective of correspondence by means of motions affirmation is to give an effective and perfect component to record motion based correspondence into words or discourse so that coordinated effort among hard of hearing and other humankind can be more sensible. Motion acknowledgment fixated on representation additionally has transform into one of the comprehensively investigation subject. Not too sharp individuals depend on gesture based communication translators for interchanges. Be that as it may, finding experienced and qualified translators for their everyday undertakings all through life period is an exceptionally troublesome errand furthermore unreasonably expensive [6]. Subsequently, human - PC communication framework will turn out to be a solid and reliable solution for such people

The expanding enthusiasm for motion examination is halfway because of the advancement of minimal effort sensors, for which there are Software Development Kits that give the way to make applications with various levels of multifaceted nature [7, 8]. Signal investigation is critical for various application fields, since it is worried with the communications amongst people, and amongst people and nature or machines [9]. These applications for the most part require the improvement of strategies for distinguishing developments made by clients, recognizing the 
fragments of the development that worry the application and perceiving the development inside a scope of expected developments [10]. With a specific end goal to fabricate these applications, distinctive systems can be utilized for tackling each previously mentioned assignment $[11,12]$. Various systems have been represented for signal arrangement and acknowledgment which could be suited for instructing show to humanoid robot. One conspicuous approach portrays the vision based acknowledgment method to accomplish visual data as highlight vector [13].

According to the theories of Gesture Recognition, it is a typical practice to record recordings of individuals conveying, through oral or communication through signing, and after that break down the motions that they make in these recordings $[14,15]$. In directing this examination, it is important to decipher a few elements of the video, for example, discourse, signal and its graphic components, stance and outward appearance, playing out an undertaking otherwise called video comment. One phase of signal interpretation is the motion stage division, which is normally performed physically by experts and is a relentless assignment [16]. Since various people have distinctive hands and fingers in physical measurement, a similar type of a motion made by two distinct people may create nonidentical numerical information when the motions are measured by normal detecting gadgets $[17,18]$. Normally, some type of changes might be required for highlight acknowledgment. Indian Sign Language exhibits static and additionally dynamic signal separately and to remember them needs to tackle a few sorts of difficulties like two gave, both hands moving a few circumstances, at times one hand is moving quick another moderate, distinctive hand shapes, reaching the body $[29,30]$.

The prevailing worldview gets thoughts from discourse acknowledgment to receive Hidden Markov Models (HMMs) for perceiving signs, which depends on the supposition that HMMs are compelling for taking care of element data with variable fleeting lengths [19]. In spite of the fact that, HMMs-based methodologies have been shown to be successful for SLR undertaking, they experience the ill effects of the issue that broad preparing to obtain the basic fleeting moves for arrangement is required, which for the most part needs a lot of preparing recordings covering varieties crosswise over various SL handlers.

The proposed framework focuses more on the effective recognition of sign gestures with the help of HMM and provides better results than the other models like Neural Networks. Adaptive Histogram technique helped in better segmentation of the input images along with Wiener Filter for removing noises in the input image. Our framework also supports in selecting and recognising the multiple hand gestures.

The remaining sections of the paper are structured with the Literature review as our 2 nd section. Third section describes our Proposed Methodology for Gesture Recognition using Segmentation method as Adaptive Histogram Technique and Features will be extracted by using Histogram Oriented Gradient (HOG) and finally HMM will be used for training the gestures and recognition process. Fourth and fifth section gives the experimental results with the suitable figures, conclusion and future work.

\section{Literature Review}

In [19] authors exhibited a system for SLR based on boosting discriminative blends of Sign Language subunits and spatiotemporal components and also developed a completely programmed framework that begins with separating signs into sensible subunits. Though, authors have not shown Sign Language translation to the respective devices, Joint learning strategy doesn't applicable to all the subunits of the sign classes.

Tamer Shanableh et.al [20] have presented numerous techniques in the gestures of Arabic Sign Language for Spatio-temporal feature extraction along with the applications in offline and online recognitions. They showed various results of classifying data with HMM and temporal dependencies. Tamer have proposed feature extraction scheme with Bayesian classifiers and $\mathrm{KNN}$ been found to yield comparable results to the more elaborate HMM based system. Authors have not shown clearly about the capability of userindependence. The test comes about have uncovered the proposed strategy gives the necessities of the calculation and capacity of the classifier as the components are exact and compact.

Shao - $\mathrm{Zi} \mathrm{Li}$ et.al [21] proposed a feature learning method for finger-spelling recognition in American sign language(ASL) which is combined Sparse Auto Encoder (SAE) with Conventional Neural Network and multiple layers PCA form a hierarchical model. Authors have proposed model with two major sections. First one gives the features to be learned from the RGB and the depth channels. The other one shows from the multiple layer PCA of features. However, the results of the system were unable to show the accurate results in terms of recognition and it's because of Sparse Auto Encoder and multiple layers. 
In [22], Galka et al. discussed on the assessment of the arrangement exhibits the aftereffects of the motion acknowledgment endeavour by utilizing a chose set of communication through signing motions with a depicted technique in light of HMM and Parallel HMM approaches. Worldly models can be thought to be more qualified to the inconstancy of development because of the high effectiveness of acknowledgment on account of inertial development parameters. Authors have not expressed completely on the exhibited framework which can be utilized to enhance the viability of vision frameworks for the impaired people.

Muhammad Rizwan et.al [23] preserved temporal context with the help of multiscale local part. DSLR system that have showed in the proposed system can makes predictions about missing gestures. They adopted 3-D histograms for gradient orientation descriptor to represent features and conducted the classification for dynamic hand gestures with the help of BOFs and Support Vector Machine. Authors have shown the experimental results only with a few gestures using DSLR system in the proposed system. The system didn't show many of the major gestures of human.

In [24], Rajeshree et.al offered a new method for sign language word recognition with 2-fold. By giving input as a video, the system can recognize any spelled word or alphabetic sequence signed in American Sign Language. The whole process consists of three major steps like recognition and detection. However, hand detection for small sentence recognition have not shown in the proposed method.

Sign language customs a transmission network among the deaf; robotic gesture recognition could more enlarge their communication with the listeners. Kosmidou and Hadjileontiadis [13, 14] have examined data from five-channel apparent electromyogram and 3-D accelerometer from the signer's prevailing hand were examined using intrinsic mode entropy (IMEn) for the mechanical acknowledgment of Greek sign language (GSL) isolated signs. Discriminant examination was used to recognize the actual measures of the central-mode purposes and the opening length for the scheming of the IMEn that subsidizes to the effective arrangement of the GSL signs. This offers an encouraging bed-set near the mechanized GSL gesture acknowledgment. Nevertheless, the intrinsic mode entropy didn't provide the effective recognition rate for multiple hand gestures.

\section{Proposed Methodology}

Gesture based communication is the normal dialect utilized by the hard of hearing individuals for correspondence. Despite the fact that they properly speak with each other by utilizing Sign Language, they defy numerous issues when they endeavour to chat with people who can listen to, especially the individuals who don't know in Sign Language it resembles perceiving the nearness of a concealed correspondence flood. Subsequently, a powerful method would be set up to accomplish and distinguish the sign motion dialects. In our anticipated exertion, we have arranged a technique for analysing and recognizing the sign motion language. Here commitments are assigned from different exertion bases.

Our anticipated technique focuses at associating transmission intermissions among the hard of hearing gathering and other individuals. Initially the noise of an image can be eliminated by Wiener Filter and the same will be segmented with the Adaptive Histogram Technique. Features like colour, pixel of a segmented image can be extracted by Multi Linear Principle Component Analysis and finally HMM is used to recognize the gestures. The detailed workflow of our proposed system has shown in the Fig 1.

\subsection{Noise removal using Wiener filter}

The backwards sifting is a rebuilding procedure for deconvolution, i.e., when the picture is obscured by a known low pass channel, it is conceivable to recuperate the picture by reverse separating or summed up converse sifting. Be that as it may, converse separating is exceptionally delicate to added substance clamour. The approach of diminishing one corruption at once permits us to build up a reclamation calculation for every sort of debasement and just consolidate them. The Wiener separating executes an ideal trade-off between converse sifting and commotion smoothing. It evacuates the added substance commotion and modifies the obscuring at the same time.

The Wiener Filter issue has answers for three conceivable cases: one where a non-causal channel is adequate (requiring an unbounded measure of both past and future information), the situation where a causal channel is wanted (utilizing a limitless measure of past information), and the limited drive reaction (FIR) situation where a limited measure of past information is utilized. 


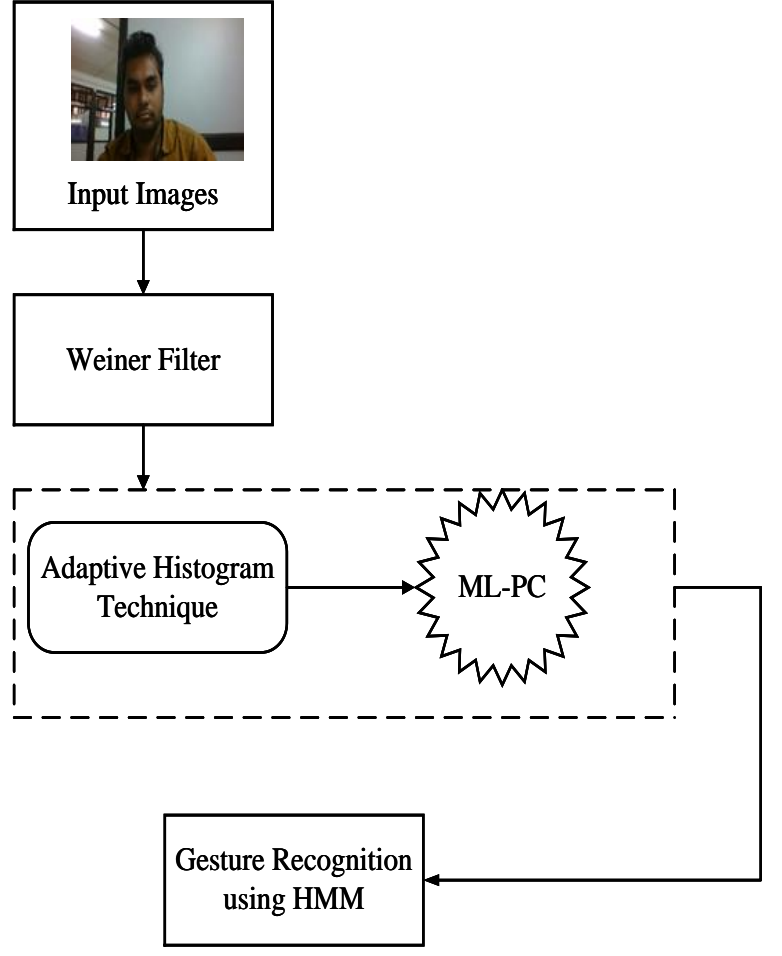

Figure.1 Workflow of Proposed framework

The principal case is easy to fathom yet is not suited for ongoing applications. Wiener's primary achievement was illuminating the situation where the causality necessity is basically and which has shown in the below equations.

$$
G(s)=S_{x, s}(S) / S_{x}(S) e^{\alpha s}
$$

\section{where $S$ - Spectral Densities}

If $G(t)$ is optimal then the minimum mean square error equation will reduce to

$$
E\left(e^{2}\right)=R_{S}(0)-\int_{-\infty}^{\infty} g(\tau) R_{x, S}(\tau+\alpha) d \tau
$$

Wiener Filter will work effectively with discrete series for finite impulse responses. The causal limited motivation reaction in Wiener channel, rather than utilizing some given information network $X$ and yield vector $Y$, finds ideal tap weights by utilizing the measurements of the info and yield signals. As per the Eq. (1) and Eq. (2), Wiener Filter populates the info lattice $X$ with assessments of the auto-connection of the information flag $(T)$ and populates the yield vector $Y$ with evaluations of the cross-relationship between the yield and information signals $(V)$.

To determine the coefficients of the Wiener channel, consider the flag $w[n]$ being nourished to a Wiener channel of request $N$ and with coefficients $\left\{x_{0}, \ldots \ldots \ldots \ldots x_{N}\right\} . a[n]$ can be represented as the output filter and the expression is as follows.

$$
a[n]=\sum_{i=0}^{N} x_{i} w[n-i]
$$

The Mean-squared Methods utilizes the way that the Wiener Filter is one that depends on the slightest squared rule of the Eq. (3), i.e. the channel minimizes the mistake between the real yield and the coveted yield. To wipe out commotion, this channel works by slender the width of the conveyance bend of Fig 2.

\subsection{Adaptive Histogram Technique for Image Segmentation}

Among number of image process techniques, Adaptive Histogram Equalization (AHE) is a popular processer method which is used to increase the contrast in a specified image. Enhancement is a typical procedure in todays advanced items. It heightens the vision ways of life as well as likewise demonstrates the ill-defined data of a picture [25]. One of the most prevalent upgrade calculations is the histogram adjustment.

The versatile division histogram upgrade or adaptive segmentation histogram enhancement (ASHE) contains three stages. The initial step is a programmed division calculation in view of discriminant investigation. The division calculation can fragment the histogram of a picture into various bunches as indicated by the grouping of the dark estimations of the prepared picture consequently.

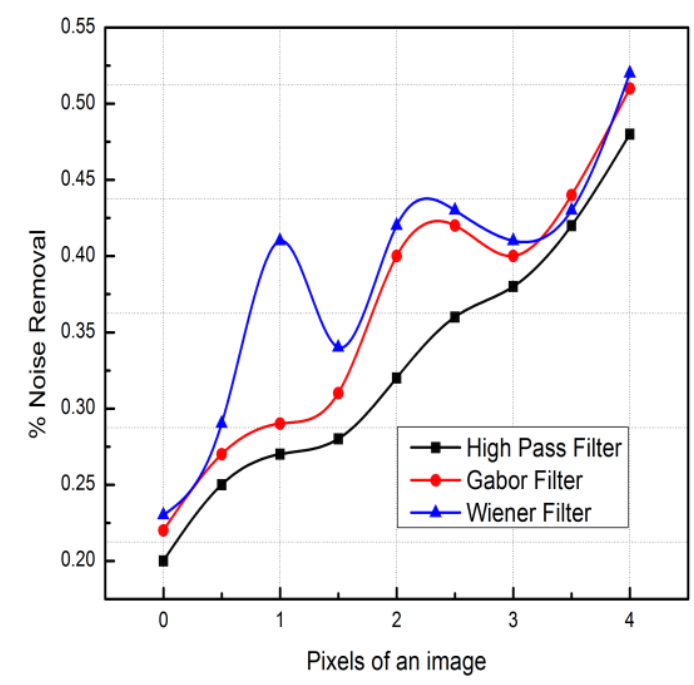

Figure.2 Percentage in various Noise Removal Techniques

DOI: $10.22266 /$ ijies2017.0630.02 
After the division calculation, distinctive question and foundation parts are sectioned into isolate bunches, called protest planes. The following stride maps the dynamic scope of the dim estimations of every bunch into a new one. The new element range is balanced by the visual attributes of every bunch. The last stride upgrades the stands out of every group from the new element extend separately. The subtle elements of the means are portrayed as taking after.

The initial step of ASHE is the programmed division calculation. In this subsection, a programmed division calculation is acquainted with fragment pictures. The calculation is an unsupervised and programmed one to segment the picture from the histogram data. For a given dark picture $Y$, the pixels of $Y$ can be apportioned into reasonable number of bunches.

The number of $k$ clusters with the $Y$ label pixels on applying thresholds from $T_{1} \ldots T_{k-l}$. Hence the clusters from $\mathrm{S}_{0}, S_{1} \ldots . S_{k+1}$ are shown below.

$$
\begin{gathered}
S_{0}=\left\{X, X+1, \ldots T_{1}\right\} \\
S_{k-1}=\left\{T_{k-1}+1, T_{k-1}+2 \ldots . L-1\right\}
\end{gathered}
$$

From the above, Eq. (4) and Eq. (5), the grey values of an image are defined by the vectors $[X$, $L-1]$ and it based on the pixel values of the $Y$ coordinates.

\subsection{Feature extraction using Multi Linear Principle Component Analysis}

Once the picture division utilizing MFCM is done, the following stride in our proposed technique is the component extraction where diverse elements for preparing the picture are extricated. Here the feature vectors containing information about the aspect ratio, holo entropy, texture based features etc. are extracted.

A run of the mill tensor question in example acknowledgment or machine vision applications is regularly indicated in a high-dimensional tensor space. Acknowledgment strategies working straightforwardly on this space experience the ill effects of the supposed revile of dimensionality [26, 27]. Taking care of high-dimensional specimens is computationally costly also, numerous classifiers perform ineffectively in high-dimensional spaces given a little number of preparing tests.

Principle Component Analysis (PCA) is an outstanding unsupervised straight method for dimensionality decrease. The focal thought behind
PCA is to decrease the dimensionality of an information set comprising of a bigger number of interrelated factors, while holding however much as could be expected the variety introduce in the first information set.

\subsection{Classification and Recognition using HMM}

Hidden Markov Model has been effectively connected to SLR frameworks to accomplish the promising execution. A Hidden Markov model can be viewed as a speculation of a blend model where the concealed factors, which control the blend part to be chosen for every perception, are connected through a Markov procedure as opposed to free of each other. In easier Markov models, the state is straightforwardly unmistakable to the spectator, and in this way the state move probabilities are the main parameters.

In a HMM, the state is not specifically obvious, but rather the yield, subject to the state, is unmistakable. Every state has a likelihood dissemination over the conceivable yield tokens. In this way, the arrangement of tokens produced by a HMM gives some data about the grouping of states. The total parameter set of a HMM can be communicated in a minimum way as follows

$$
\varphi=(V, O, \pi)
$$

where

$V$ - state transition matrix

$O$ - observation probability matrix

$\pi$ - initial probability distribution of states

The Gaussian blend based demonstrating as delineated in the past subsection, speaks to a strong method for evaluating the PDF for every movement design class [26]. This strategy can be useful in demonstrating classes where substance is timeinvariant and try not to have a solid reliance on worldly requesting. Our sub trajectory-based representation approach models the directions as an arrangement of sub trajectories. This approach requires a plan that takes the worldly reliance among sub trajectories into record. We propose to embrace the utilization of well for direction grouping and acknowledgment applications. Well permit the framework to remain in a similar state on the other hand to travel to the following state at any given time as indicated by state move probabilities learnt from preparing information. This permits demonstrating of worldly varieties, where the term of the state is a variable. 
In this specific situation, we are keen on demonstrating a class of protest movements in view of the fleeting requesting of sub trajectories. Since sub trajectories speak to sections of nuclear movements between purposes of progress in movement design, the subsequent procedure can be demonstrated as first order Markov chain. We likewise watch that blend of Gaussians is a vigorous technique for evaluating the PDF in the nonattendance of transient varieties. We, in this manner propose to utilize ceaseless thickness HMMs, where every condition of the HMM is demonstrated by a blend of Gaussians. So as to endeavour saliency requesting when ordering pictures, we propose to factually show the image changes in a roundabout way by considering them as perceptions related to a chain of obscure (concealed) states, which can be depicted as a HMM.

The principal parameter indicated for a HMM is the quantity of states. For every class, spoke to by a different HMM, we set the quantity of states equivalent to the most extreme number of sub trajectories in all the preparation set directions for that class. Once the quantity of states is altered, the total arrangement of model parameters portraying the HMM are given by the triplet and which is shown in the below equation.

$$
\delta=\left\{a_{i j}, b_{j}, \pi_{j}\right\}
$$

where

$a_{i j}$ - denotes probability of $j^{\text {th }}$ subtrajectory

$b_{j}$ - denotes the input state.

\section{Results and Discussions}

Data Acquisition and Recognition for the proposed Sign-Gesture Language is represented in the working stage of MATLAB. The acknowledgment procedure is tried with casings of various recordings and the got aftereffect of the proposed work has been demonstrated. At first, the video is fragmented to various shots or casings and afterward components are removed trailed by the acknowledgment procedure.

As stated in the section 3, for every data outlines the different component are extricated utilizing the calculations specified as a part of the proposed strategy. These components help in distinguishing the words in every casing. Once the elements are extricated, the accurate words are perceived. Fig 3 and Fig 4 demonstrates the outcomes got by the proposed strategy.

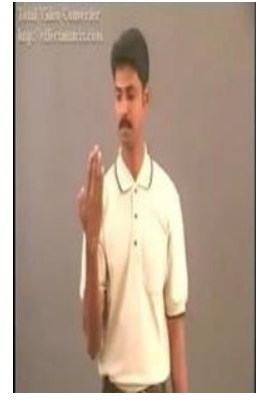

(a)

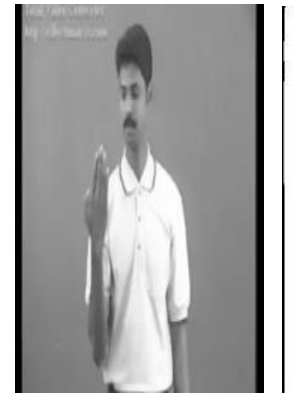

(b)

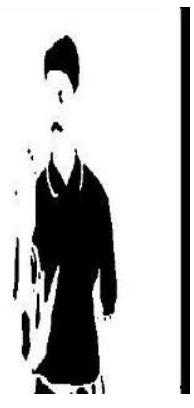

(c)
Figure.3 Sign Gesture Recognition for 'Green'

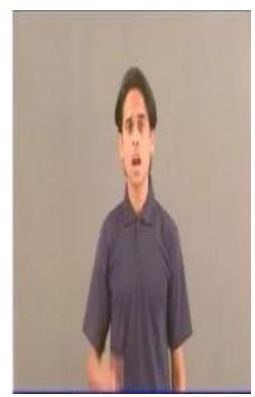

(a)

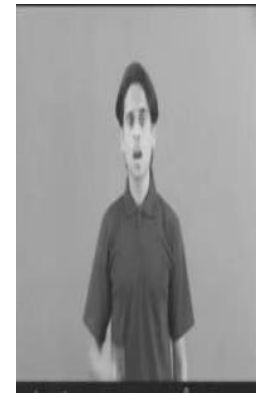

(b)

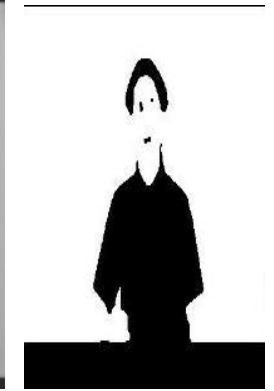

(c)
Figure.4 Sign Gesture Recognition for 'Lunch'

The Fig 3 shows the sign gesture recognition for the colour 'Green'. Fig 4 (a) is the input frame of which is to be processed. Fig 4(b) shows the noise removed output of the input frame. Fig 4(c) is the segmented output which is obtained from noise removed output. Based on this, the classification of the sign gesture is done once the features are extracted. Similar outputs for 'Green' and 'Lunch' are shown in Fig 3 and Fig 4 respectively.

The intrinsic difficulties of perceiving communication via gestures signals can be outlined as takes after - Algorithms of Recognition should work for an expansive, unclosed set of words. Segmentation methods should distinguish the various hand movements of a complex system. Motion acknowledgment must be pivot invariant to bolster viable executions.

Every picture conveys distinctive words as sign motion and these pictures in the wake of handling through the diverse strides in our proposed strategy the motion are at last perceived. Figure 4 given underneath demonstrates the perceived words from various data sources. The got comes about exhibit that the proposed methodology passes on best affirmation of sign movement over other existing procedures. 
The execution estimation of our proposed rationality is found out by measuring the exactness of the system. The precision of our proposed technique turned out to be more effective than the current works. The current calculation which we think about here is the neural system. The normal affirmation rate got in our proposed procedure has better outperformed when differentiated and these present systems. The Table 1 given underneath shows the affirmation rate obtained from our proposed system and the present methodology. The framework is tried utilizing the component values from the set of input images. The acknowledgment rate of the framework is assessed as the proportion of the quantity of signs distinguished accurately to the aggregate number of signs in the test dataset.

Our Proposed method is well suitable for gesture recognition compared to the conventional methods like neural networks [28] and support vector machine [8]. Previous frameworks [8, 28] were not enough in removing noise from the input images, hence we have achieved better results for noise removal using Wiener Filter. We have used Adaptive Histogram technique for image segmentation and completed segmentation process in very less time. Our enhanced framework mainly attains the better recognition rate compared to the previous methods in $[8,28]$.

In [28], authors have developed a system for Recognition of Sign Gestures using Modified Region Growing algorithm and Adaptive Fuzzy Classifier and which had shown appropriate results than the conventional classifiers like NN, SVM, hence they got the average recognition rate as $83 \%$. In this paper, we got better results in terms of feature extraction by Multi Linear PCA and classification of gestures has been carried out by Hidden Markov Model using possible input and output states of the recogniser. Extracted features from the input images are trained to the HMM classifier to recognize the signs. Using HMM we have achieved average recognition rate as 84 percentage.

From the below table, doubtlessly our proposed methodology passes on favoured affirmation rate over the existing strategy $[8,28]$. The relating graphical representation of the affirmation rate is showed in the below figure. The results demonstrate that our arranged arrangement of sign motion acknowledgment is productive in perceiving the correct words from the sign motion and can successfully help in connecting the correspondence hole between the hard of hearing group with the customary people.
Table 1. Average Recognition rate for proposed HMM method and remaining methods

\begin{tabular}{|c|c|}
\hline Method & $\begin{array}{c}\text { Average } \\
\text { Recognition Rate } \\
(\%)\end{array}$ \\
\hline $\begin{array}{c}\text { HMM } \\
\text { (Proposed method) }\end{array}$ & 84 \\
\hline Neural Network & 79 \\
\hline $\begin{array}{c}\text { Support Vector } \\
\text { Machine }\end{array}$ & 72 \\
\hline
\end{tabular}

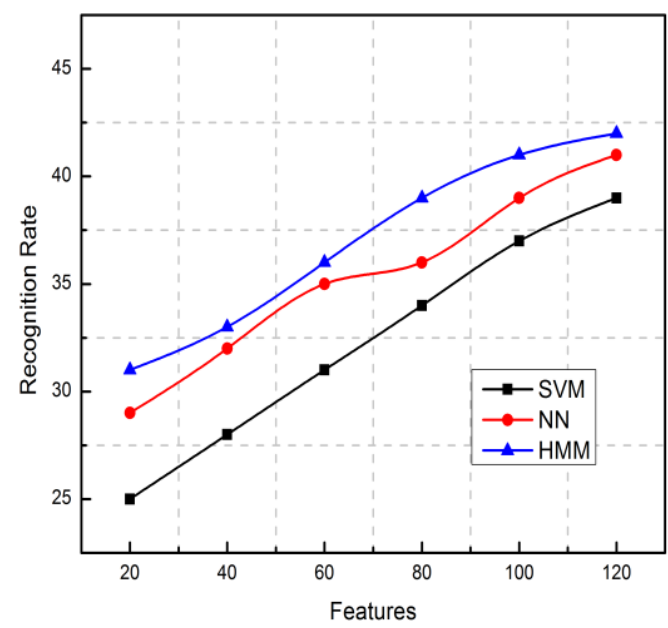

Figure.5 Recognition rate in various classifiers

\section{Conclusion}

In our work, we have proposed a beneficial methodology for Sign motion securing and acknowledgment utilizing altered locale developing calculation using HMM and AHT and achieved better results as average recognition rate of $84 \%$. The sign motion frames a fundamental method of correspondence with the hard of hearing group and its securing and acknowledgment assumes an imperative part for the correspondence with the individuals who are new to sign signals. Our proposed strategy helps in perceiving every last motion with higher precision and easily. We utilized element extraction organize alongside the division which frames an effective procedure in extricating the required measures for acknowledgment. This is trailed by HMM based order handle which conveys the correct perceived yield from the sign motion. 
The consequences of our technique demonstrate that it is more effective when we contrast with other existing works related with sign motion acknowledgment.

The outcomes of our strategy demonstrate that it is more effective when we contrast with other existing works related with sign motion acknowledgment. In future, we plan to work for a large number of signs particularly for the signs of kids to understand the inner feelings and responses of them.

\section{References}

[1] S. Mitra and T. Acharya, "Gesture Recognition: A Survey", IEEE Transactions on Systems, Man, and Cybernetics, Part $C$ (Applications and Reviews), Vol.37, No.3, pp. 311-324, 2007.

[2] R. Yan and K. Peng Tee, "Gesture Recognition Based on Localist Attractor Networks with Application to Robot Control", IEEE Journal of Computational Intelligence Magazine, Vol.7, No.1, pp.64-74, 2012.

[3] C. Zhu and W. Sheng, "Wearable Sensor-Based Hand Gesture and Daily Activity Recognition for Robot-Assisted Living", IEEE Transaction on System, Man and Cybernetics Part A: Systems and Humans, Vol.41, No.3, pp.567-573, 2011.

[4] R. Kaluri, P. Reddy Ch, "An Overview on Human Gesture Recognition", International Journal of Pharmacy and Technology, Vol.08, No.04, pp.12037-12045, 2016.

[5] J. Wang and F. Chuang, "An AccelerometerBased Digital Pen with a Trajectory Recognition Algorithm for Handwritten Digit and Gesture Recognition", IEEE Transaction on Industrial Electronics, Vol.59, No.7, pp.2998-3006, 2012.

[6] C. Tran and M. Manubhai Trivedi, "3-D Posture and Gesture Recognition for Interactivity in Smart Spaces", IEEE Transaction on Industrial Informatics, Vol.8, No.1, pp.178-187, 2012.

[7] G. Fang, W. Gao and D. Zhao, "Large Vocabulary Sign Language Recognition Based on Fuzzy Decision Trees", IEEE Transactions on Systems, Man, And Cybernetics-Part A: Systems and Humans, Vol.34, No.3, pp.305-314, 2004.

[8] R. Kaluri and P. Reddy Ch, "A framework for sign gesture recognition using improved genetic algorithm and adaptive filter", Cogent Engineering, Vol.3, pp.1-9, 2016.

[9] L. TzuuHseng, K. MinChi and P. Kuo, "Recognition System for Home-Service-Related Sign Language Using Entropy-Based K -Means
Algorithm and ABC-Based HMM", IEEE Transactions on Systems, Man, and Cybernetics: Systems, Vol.46, No.1, pp.150-162, 2015.

[10]R. Yang, S. Sarkar and B. Loeding, "Handling Movement Epenthesis and Hand Segmentation Ambiguities in Continuous Sign Language Recognition Using Nested Dynamic Programming", IEEE Transactions on Pattern Analysis and Machine Intelligence, Vol.32, No.3, pp.462-477, 2010.

[11]B. Yi, X. Wang, F. C. Harris and S. M. Dascalu, "sEditor: A Prototype for a Sign Language Interfacing System", IEEE Transactions on Human-Machine Systems, Vol.44, No.4, pp.499510, 2014.

[12] Y. Yao and Y. Fu, "Contour Model based HandGesture Recognition using Kinect Sensor", IEEE Transaction on Circuit and System for Video Technology, Vol.24, No.11, pp.1051-8215, 2014.

[13] V. E. Kosmidou and L. J. Hadjileontiadis, "Sign Language Recognition Using Intrinsic-Mode Sample Entropy on sEMG and Accelerometer Data", IEEE Transactions on Biomedical Engineering, Vol.56, No.12, pp.2879-2890, 2009.

[14] V. E. Kosmidou, P. C. Petrantonakis and L. J. Hadjileontiadis, "Enhanced Sign Language Recognition Using Weighted Intrinsic-Mode Entropy and Signer's Level of Deafness", IEEE Transactions on Systems, Man, And Cybernetics-Part B: Cybernetics, Vol.41, No.6, pp.1531-1543, 2011.

[15]A. Akl and C. Feng, "A Novel AccelerometerBased Gesture Recognition System", IEEE Transaction on Signal Processing, Vol.59, No.12, pp.6197-6205, 2011.

[16]N. Tubaiz, T. Shanableh and K. Assaleh, "Glove-Based Continuous Arabic Sign Language Recognition in User-Dependent Mode", IEEE Transactions on Human-Machine Systems, Vol.45, No.4, pp.526-533, 2015.

[17]D. Frolova and H. Stern, "Most Probable Longest Common Subsequence for Recognition of Gesture Character Input", IEEE Transaction on Cybernetics, Vol.43, No.3, pp.871-880, 2013.

[18]H. Cheng and J. Luo, "A Windowed Dynamic Time Warping Approach for 3rd Continuous Hand Gesture Recognition", In: Proc. of IEEE International Conference on Multimedia and Expo (ICMP), China, pp.1-6, 2014.

[19]H. Junwei, G. Awad and A. Sutherland, "Boosted subunits: a framework for recognising sign language from videos", IET Image Processing, Vol.7, No.1, pp. 70-80, 2013. 
[20]T. Shanableh, K. Assaleh and M. Al-Rousan, "Spatio-temporal feature-extraction techniques for isolated gesture recognition in Arabic sign language", IEEE Transactions on Systems, Man, and Cybernetics, Part B (Cybernetics), Vol.37, No.3, pp.641-650, 2007.

[21]S. Z. Li, B. Yu, W. Wu, S. Z. Su and J. R. R "Feature learning based on SAE-PCA network for human gesture recognition in RGBD images", Neurocomputing, Vol.151, pp.565-573, 2015.

[22]J. Gałka, M. Mąsior, M. Zaborski and K. Barczewska, "Inertial Motion Sensing Glove for Sign Language Gesture Acquisition and Recognition", IEEE Sensors Journal, Vol.16, No.16, pp.6310-6316, 2016.

[23]M. R. Abid, E. M. Petriu and E. Amjadian, "Dynamic sign language recognition for smart home interactive application using stochastic linear formal grammar", IEEE Transactions on Instrumentation and Measurement, Vol.64, No.3, pp.596-605, 2015.

[24]R. S. Rokade and D. Doye, "Spelled sign word recognition using key frame", IET Image Processing, Vol.9, No.5, pp.381-388, 2014.

[25]R. Kaluri "A comparative study on Image Segmentation Techniques", International Journal of PT, Vol.8, No.2, pp.12712-12717, 2016.

[26]B. Bashir, I. Faisal, A. Khokhar and D. Schonfeld. "Object trajectory-based activity classification and recognition using hidden Markov models", IEEE transactions on Image Processing, Vol.16, No.7, pp.1912-1919, 2007.

[27]H. Lu, K. N. Plataniotis and A. N. Venetsanopoulos, "MPCA: Multilinear principal component analysis of tensor objects", IEEE Transactions on Neural Networks, Vol.19, No.1, pp.18-39, 2008.

[28]R. Kaluri, P. Reddy Ch, "Sign Gesture Recognition using Modified Region Growing Algorithm and Adaptive Genetic Fuzzy Classifier." International Journal of Intelligent Engineering and Systems, Vol.9, No.4, pp.225233, 2016.

[29]L. Kuruva, N. Khare, "Constraint-Based Measures for DNA Sequence Mining using Group Search Optimization Algorithm", International Journal of Intelligent Engineering and systems, Vol.9, No.3, pp.91$100,2016$.

[30]G. T. Reddy, N. Khare, "FFBAT-Optimized Rule Based Fuzzy Logic Classifier for Diabetes", International Journal of Engineering Research in Africa, Vol.24, No.2, pp.137-152, 2016. 